\title{
RADIO SOURCES MODELLING AND EMISSION MECHANISMS (DISCUSSION)
}

Discussion of the paper presented by MELROSE (p. 423)

Perlman: One of the results from my own observations of PKS $1413+135$, one extragalactic flat-spectrum source (which unfortunately is not part of my poster) is that the angular size of the inverted-spectrum core at 13 and $18 \mathrm{~cm}$ is in fact proportional to $1 / \nu$. I can show you the data later.

Melrose: I am interested to see your data.

Cotton: In Cotton et al. 1980 the flat-spectrum source $0735+178$ was observed interferometrically and decomposed into a number of components which were each well represented by a simple, synchrotron model where each component had a peaked spectrum but which added to a flat spectrum. This lead to the term "cosmic conspiracy" as the components "know" about each other.

Melrose: My remarks on the "cosmic conspiracy" refer to the specific model in which the flux density depends only on the product of frequency and radius. I do not believe this mode; can be the correct explanation for flat spectra.

Dogiel: The spectrum generated by multiple shock acceleration strongly depends on the age of an emitting object. As it was shown by Bynol and Toptygin for the case of $\mathrm{OB}$-associations, there is an energy range where the spectrum is $E^{-1}\left(E<E_{0}\right)$ and at higher energies $E>E_{0}$ the spectrum of electrons is very steep. The position of the break $E_{0}$ is a function of time, $E_{0}=E_{0}(t)$.

Melrose: I agree. There is a high energy portion of the energy spectrum whose spectral index is determined by the compression ratio of the shocks. The hard-spectrum with $a \approx 1$ forms at low energy and moves to higher energy with increasing number of encounters with shocks.

Pohl: Inverted non-thermal emission is not only observed from the filaments but also from the diffuse region in the arc, where the B-field is 
much lower. Would your process also work there?

Melrose: I am aware of the observations to which you refer, and my model does not explain such diffuse emission in a natural way. I shall be interested to see whether this observation of diffuse emissions is confirmed.

Pohl: AGN radio outbursts have in the optically thin part of the spectrum an electron spectral index of more than $1(\alpha>0)$. On the other hand, in view of the high synchrotron and inverse-Compton losses one would expect the blocking effect and hence the pile-up to be very strong. Isn't this contradictory?

Melrose: I don't see any contradictions. Schlickeiser (1984) has proposed a pile-up model, similar to the one I am suggesting, for optical/IR emission with an inverted spectrum. Note that high synchrotron or inverse Compton losses implies a pile-up only if it occurs at the same time and place as Fermi acceleration. No pile-up occurs for diffusive shock acceleration.

Falcke: I don't see what the GC filaments have to do with the cosmic conspiracy!? Those filaments are associated with large scale magnetic field lines and molecular clouds and are very different from the situation in the AGN. In fact, the only source similar to an AGN, $\mathrm{Sgr} \mathbf{A}^{*}$, can well be explained by a "cosmic conspiracy" theory type model. (A\&A 278,L1)

Melrose: My view is that the GC sources with flat spectra require hard electron spectra. It seems relevant to ask whether the explanation for flat spectra in the GC might not also be relevant to AGN. I am suggesting that in the absence of clear evidence for self absorption in flat spectral sources, the default explanation should be a hard electron spectrum rather than a "cosmic conspiracy".

Discussion of the paper presented by MEIER (p. 433)

Sunyaev: What is the ratio of the magnetic field pressure to the plasma pressure in the disk you assume in your computations as initial value?

Meier: Formally this ratio is infinitesimally small. The disk itself in the simulations is infinitely thin and anchors the magnetic field. The material being accelerated is the disk corona. The ratio of the magnetic pressure in the corona to the coronal gas pressure is a parameter in our models and varies from about $1 / 2$ to 2 . Note that this ratio can be even higher without the field leaving the system because of our assumption of a cold, field-anchoring thin disk below the corona. 
Begelman: Do the jets remain well collimated - solely by magnetic tension well beyond the critical surfaces, or is external confinement necessary?

Meier: Yes, the jets remain well collimated, mainly by magnetic tension, as far from the central objects as we have computed them ( $\sim 300$ gravitational radii), especially in their inner core. For high external pressures, the cocoon appears to be largely pressure confined. However, for very low pressure the cocoon itself, though much larger than in the high pressure case, appears to be magnetically confined. Note that in this mechanism, as opposed to the classical Blandford-Payne process, the critical surfaces are all very close to the disk. The material rises from the disk with trans- or super Alfvenic velocities rather than accelerating slowly through several critical surfaces over a large distance. Discussion of the paper presented by BEGELMAN (p. 441)

Romanova: How do you explain the optical and X-ray radiation of M87?

Begelman: The optical and X-ray emission from the knots is readily explained as synchrotron radiation.

Discussion of the paper presented by DE YOUNG (p. 461)

Trussoni: Is the total jump of velocity across the sheer layer subsonic or supersonic?

De Young: In this case it is subsonic. However, this may in fact be the case, since the boundary layer is between the jet and the hot post shock gas behind the bow shock. In these regions the sound speeds are very high.

Eilek: To what extent does the vortex saturation, and thus the asymptotic entrainment rate, depend on the viscosity of the fluid?

De Young: These are very high Reynolds number flows. Empirically, it appears that the complex turbulent dissipation occurs deep inside the large scale structures; hence viscosity is important there. However, the growth and saturation of the large scale structures occurs on a much larger scale and is unaffected by viscosity.

Discussion of the paper presented by FERRUIT (p. 465)

Wilson: Does your model include ionizing radiation from the bow shock or is the ionization completely dominated by photons from the AGN? 
Ferruit: No, the only ionization taken into account is from the AGN (no pre-ionization of the ISM by the shock radiation itself.)

Steffen: Do you include thermal mixing of the grid parcels as they move along the bowshock? Do you expect any significant influence of this effect?

Ferruit: No, we did not include any mixing. We can expect a significant effect in the tail where turbulence is likely to occur, but there, the temperature differences between particles are lower. Discussion of the paper presented by EILEK (p. 483)

Meisenheimer: We do see the spectral break in the lobes of M87 and it is still in the NIR/optical frequency range.

Eilek: Yes, that is an important test case for these questions.

Aller: Do we ever see true breaks in spectra?

Eilek: We do commonly see high-frequency spectral turnovers; but it's not easy to determine the exact shape of the high-frequency spectrum, where the source becomes very faint.

Aller: What about the suggestion that particle energies are redistributed in situ in shocks such that the resulting spectral index is set by the "local" jump conditions.

Eilek: That is certainly an important case of local reacceleration - although I suspect its more relevant to jets and hot spots than to the more diffuse lobes and tails.

Discussion of the paper presented by $\underline{R U D N I C K}$ (p. 489)

Ekers: Why call this "tomography"? It is not the usual meaning of the word.

Rudnick: "Tomography" comes from the Greek word "tomos", meaning a section or cut. We use the word deliberately because of its connotation of uncovering hidden features. However, to avoid confusion with the conventional usage, we call the difference maps between two frequencies "spectral tomography", while a related technique, which we introduce elsewhere, we call "polarization tomography".

Ekers: But it still has nothing to do with the process of reconstruction of an object from its "sections" or "cuts". 